\title{
Outbreak
}

\section{An outbreak of Elizabethkingia meningoseptica neonatal meningitis in Mauritius}

\author{
Mohammad I. Issack ${ }^{1}$ and Yaseen Neetoo ${ }^{2}$ \\ ${ }^{1}$ Central Health Laboratory, Victoria Hospital, Candos, Mauritius \\ ${ }^{2}$ Paediatric Unit, Jawarharlal Nehru Hospital, Rose-Belle, Mauritius
}

\begin{abstract}
Introduction: Elizabethkingia meningoseptica is a rare but well-recognised cause of neonatal meningitis. Reported outbreaks have involved very few cases. We describe the management and outcome of a relatively large outbreak of E.meningoseptica neonatal meningitis Methodology: From August 2002 to December 2003, eight cases of meningitis caused by E. meningoseptica occurred among babies admitted to the neonatal ward of Jawarhlal Nehru hospital, Mauritius. In all cases, the organism was isolated from the cerebrospinal fluid. Infection control measures were re-emphasized after each case and environmental swabs were cultured on several occasions.

Results: The affected babies were aged 6 to 20 days (mean age of 10 days). Seven of the babies weighed $<2,500 \mathrm{~g}$. All CSF isolates had the same antibiotic susceptibility pattern. Apart from one baby who died shortly after admission, all cases responded to treatment with intravenous piperacillin and oral rifampicin for three weeks. Hydrocephalus developed in two babies and was subsequently fatal in one case. At follow-up of the other cases, one baby had severe neurological sequelae but a full recovery was observed in the other four cases. The source of the outbreak could not be established conclusively.

Conclusions: The outcome was better than what has been reported in the medical literature. Prompt identification of the causative organism and initiation of appropriate antimicrobial therapy is essential. The combination of piperacillin and rifampicin should be considered an option for the treatment of E. meningoseptica neonatal meningitis if supported by properly performed antibiotic susceptibility test results.
\end{abstract}

Key words: Elizabethkingia meningoseptica; meningitis; neonatal; outbreak; Mauritius

J Infect Dev Ctries 2011; 5(12):834-839.

(Received 25 Janaury 2011 - Accepted 06 April 2011)

Copyright $\left({ }_{0} 2011\right.$ Issack and Neeto. This is an open-access article distributed under the Creative Commons Attribution License, which permits unrestricted use, distribution, and reproduction in any medium, provided the original work is properly cited.

\section{Introduction}

Elizabethkingia meningoseptica, formerly known as CDC group IIa, Flavobacterium meningosepticum and Chryseobacterium meningosepticum, is Gramnegative, rod-shaped bacterium widely distributed in nature, particularly in soil and water [1]. It was first recognized as a cause of neonatal meningitis by Elizabeth King in 1959 [2]. Subsequently, several cases of infection, especially septicemia, neonatal meningitis and adult pneumonia, as well as colonization in humans, have been described. [3].

E. meningoseptica meningitis occurs mainly in premature babies in the first two weeks of life. It is fatal in more than half of the cases and sequelae are common among the survivors [1,3]. Although E. meningoseptica meningitis usually accounts for a very small proportion of neonatal meningitis, accurate diagnosis is important because the organism is usually resistant to multiple antibiotics, including ampicillin, cefotaxime and gentamicin, largely used in the empirical treatment of this life-threatening infection.

Many cases of E. meningoseptica infections have been reported as part of outbreaks which have been attributed to contamination of hospital tap-water, disinfectants, saline, antibiotic solutions, lipid solution, sink drains and respiratory equipment $[1,4,5]$. In most reported outbreaks, the number of cases of neonatal meningitis was small and rarely exceeded five. We describe an outbreak in a neonatal ward of E. meningoseptica meningitis which affected eight babies over a sixteen-month period.

\section{Methodology}

Jawarharlal Nehru hospital (JNH) is one of the five general hospitals in Mauritius, a middle-income island nation in the Indian Ocean. In 2003, the number of babies delivered at JNH was 2,586, representing $16.3 \%$ of all hospital deliveries and $13.8 \%$ of all births in Mauritius [6]. There is one 
Table 1. Details of cases of Elizabethkingia meningoseptica meningitis

\begin{tabular}{|c|c|c|c|c|c|c|c|c|}
\hline Case & $\begin{array}{c}\text { Specimen } \\
\text { date }\end{array}$ & Sex & $\begin{array}{c}\text { Birth weight } \\
(\mathrm{kg})\end{array}$ & D.O.B & $\begin{array}{c}\text { Delivery } \\
\text { mode }\end{array}$ & $\begin{array}{c}\text { Age } \\
\text { (days) }\end{array}$ & $\begin{array}{c}\text { Organisms on Gram- } \\
\text { stain of CSF }\end{array}$ & Outcome \\
\hline 1 & 09.08 .02 & Female & 2.3 & 31.07 .02 & CS & 9 & Not seen & Full recovery \\
\hline 2 & 25.11 .02 & Male & 2.4 & 16.11 .02 & NVD & 9 & Few & Full recovery \\
\hline 3 & 05.03 .03 & Female & 1.6 & 13.02 .03 & CS & 20 & Scanty & Cerebral palsy \\
\hline 4 & 08.06 .03 & Male & 2.2 & 02.06 .03 & NVD & 6 & Many & Died \\
\hline 5 & 27.06 .03 & Female & 2.0 & 14.06 .03 & NVD & 13 & Few & Hydrocephalus, died \\
\hline 6 & 19.07 .03 & Female & 2.2 & 12.07 .03 & CS & 7 & Many & Hydrocephalus, recovered \\
\hline 7 & 03.10 .03 & Female & 2.9 & 26.09 .03 & CS & 8 & Moderate & Full recovery \\
\hline 8 & 17.12 .03 & Female & 1.3 & 10.12 .03 & NVD & 7 & Scanty & Full recovery \\
\hline
\end{tabular}

neonatal ward at JNH for newborns. Between August of 2002 and December of 2003, eight neonates who had stayed in that ward at JNH were diagnosed with E. meningoseptica meningitis. At that time, it had been routine practice to admit to the neonatal ward all newborn babies except for those born by normal vaginal delivery and weighing $>2,500 \mathrm{~g}$. The latter were admitted to the post-natal ward.

All neonates with suspected sepsis had blood cultures collected prior to starting or changing antibiotics. In all cases of possible meningitis and most cases of sepsis of uncertain origin, lumbar puncture was performed for cerebrospinal fluid (CSF) examination.

All CSF specimens were cultured on Columbia blood agar in air and under anaerobic conditions, on Cysteine Lactose Electrolyte Deficient (CLED) agar, and on chocolate agar in carbon dioxide. $E$ .meningoseptica was identified by Gram-stain appearance, non-lactose fermentation on CLED agar, positive cytochrome oxidase reaction and API 20NE (Bio-Mérieux, Marcy l'Etoile, France). Antibiotic susceptibility testing was performed by disc diffusion on Mueller-Hinton agar and interpreted by the Stokes method [7].

After confirmation of seven of the eight cases, every case of E. meningoseptica meningitis, environmental screening was conducted in the neonatal ward. In one instance, environmental specimens were also sent from the labour ward. Screening of other asymptomatic babies in the neonatal ward was also conducted on one occasion with culture of rectal and umbilical swabs. A total of 136 specimens were received between August 2002 and November 2003, including incubator water (58), oxygen humidifier water (24), suction fluid (23), disinfectants (12) and tap water (5) and 14 miscellaneous specimens.
Screening specimens were cultured on CLED agar and E. meningoseptica was identified as described above.

Infection control measures were reviewed after confirmation of each case of E. meningoseptica neonatal meningitis.

\section{Results}

E. meningoseptica was isolated from the CSF of eight neonates. Details of the cases are shown in Table 1. Six of the eight cases $(75 \%)$ were born after less than 37 weeks' gestation. Seven of the affected babies $(88 \%)$ weighed $<2,500 \mathrm{~g}$ at birth. The babies were aged 6 to 20 days with a mean of 10 days and a median age of 8.5 days. All but one were aged less than two weeks. Three of the neonates were admitted with meningitis after having been discharged home from the neonatal ward whereas the other five had been in the ward from birth until they developed meningitis. During the same period, no case of $E$. meningoseptica meningitis was recorded in Mauritius from any other health-care institution.

The E. meningoseptica isolates were all susceptible to piperacillin, piperacillin/tazobactam, vancomycin and rifampicin and resistant to cefalexin, ceftazidime, cefotaxime, ceftriaxone, gentamicin, amikacin, tetracycline, colistin and meropenem. They had intermediate susceptibility to ciprofloxacin and amoxicillin/clavulanic acid.

One baby died two hours after admission. The seven other babies were treated for three weeks with intravenous piperacillin $(100 \mathrm{mg} / \mathrm{kg}$ every 8 hours $)$ and oral rifampicin (10 mg/kg every 12 hours) via a nasogastric tube after the isolation of $E$. meningoseptica was confirmed. In cases 5 to 8 , piperacillin had already been added to the empirical treatment when Gram-negative bacilli were seen on the Gram-stain of CSF. Rapid defervescence occurred in all cases with clinical improvement. 
However, two cases developed hydrocephalus and required insertion of a ventriculoperitoneal shunt. One of them subsequently died seven months later with complications of hydrocephalus whereas the other one remained healthy with normal developmental milestones three years later. One other baby was subsequently diagnosed with cerebral palsy secondary to the E. meningoseptica meningitis. The other four babies did not develop any complication and had remained well without any neurological sequelae noted after three years of follow-up in the out-patients clinic.

Two specimens of water from incubators were positive for E. meningoseptica and in both cases, an infected baby had been nursed in them just before the sample was obtained. On six occasions, suction fluid specimens in use in the neonatal and labour wards were positive for E. meningoseptica. A specimen of suction fluid from the operating theatre was also positive but the isolate had a different susceptibility pattern. E. meningoseptica was not isolated from the 127 other specimens, including all tap water samples.

Of eight asymptomatic babies screened, two were found to be carriers of E. meningoseptica. In one baby, both rectal and umbilical swabs were positive whereas in the second case, only the rectal swab was positive.

During the outbreak, many potentially hazardous procedures were identified and vigorous infection control measures were instituted to eliminate or reduce the risk of nosocomial infections in neonates. Incubators were partially dismantled and thoroughly cleaned and disinfected in between use for different babies. Medical and nursing staff were instructed to stop the use of multiple doses from vials of medication or fluids intended for single use only. The importance of hand disinfection in between contact with different babies was re-emphasized. In addition to new suction catheters being used for each baby as was the previous practice, suction containers and tubes were changed and disinfected more regularly, at least once daily. The admission policy to the neonatal ward was also reviewed, and more babies, especially term babies born by cesarean section, were admitted to the post-natal ward with their mother rather than in the neonatal ward.

From December 2003 to date, no case of $E$. meningoseptica meningitis has occurred in the neonatal ward of $\mathrm{JNH}$, and in Mauritius generally only two cases of have been recorded. Both the cases occurred in 2005 in neonates from another hospital.

\section{Discussion}

In the outbreak of E. meningoseptica described here, the cases occurred intermittently over a period of 16 months. Most reported outbreaks among neonates have lasted a few weeks to a few months $[5,8]$. However, in one outbreak, the same strain of $E$. meningoseptica was isolated from eight babies over almost two years though most cases were asymptomatic [4]. It has been suggested that outbreaks caused by environmental contamination tend to be intermittent [9]. However, it is more likely that E. meningoseptica has a low degree of pathogenicity and only a small percentage of colonized patients develop sepsis [3]. Thus transmission may have occurred throughout the 16month period with several neonates becoming colonized without developing any symptoms. Indeed, in some previously reported outbreaks, asymptomatic cases were detected and did not require treatment $[4,8]$. We screened asymptomatic babies at one point in time and we identified two neonates who were colonized with E. meningoseptica but remained well without antimicrobial treatment. Some types of $E$. meningoseptica may also be more likely than others to cause disease, and although serotyping of $E$. meningoseptica is rarely performed, limited data available from the literature suggests that serotype $\mathrm{C}$ is more strongly associated with neonatal infection [3].

Infection control measures are an essential part of the management of outbreaks of E. meningospetica infection to limit spread of the organism to other patients. Environmental sampling is required to attempt to identify the possible source. In some previously reported outbreaks, measures such as closing the unit to new admissions, disinfecting the unit thoroughly, and changing the choice for empiric antibiotics were taken. However, in other cases, milder measures such as use of alcoholic hand rub, toileting of babies with sterile instead of tap water, repair and chlorination of hospital water tanks, and changing of sink taps were successful in controlling the outbreaks [8].

We could not convincingly identify the source of the outbreak. Isolation of $E$. meningoseptica from water in the incubator on two occasions was probably the consequence rather than the cause of neonatal infection. Similarly, it was not clear if isolation of the organism in suction fluids was due to previous use on already colonized babies or whether it was the source of infection. One or more sources were identified in most reported outbreaks of E. meningoseptica but 
there are also reports where no source could be identified despite extensive investigation and culture of environmental specimens [8,10]. Our outbreak subsequently ended and it could have been due to one or more of the measures taken. However, it is also possible that termination of the outbreak occurred because the unidentified source was unwittingly eliminated or because for unknown reasons the conditions for persistence of the outbreak ceased to be present.

An important limitation of our report is the absence of typing data on the isolates to confirm their clonality. However, the isolation of $E$. meningoseptica with similar antibiotic susceptibility patterns from the CSF of neonates who had been in the same ward of JNH over a period when no similar case was identified from neonates admitted to other hospitals is very suggestive of an outbreak. On the other hand, determining the significance of the environmental isolates is more problematic without typing data.

The most appropriate choice of effective antimicrobial agents for the treatment of $E$. meningoseptica meningitis is difficult to determine. Early investigators recommended vancomycin but this has subsequently been questioned by other groups [3,11-14]. Discrepancies in sensitivity patterns by disc diffusion and broth dilution have been noted in some studies and treatment failures have occurred following the use of an antibiotic to which the organism was considered sensitive by disc diffusion but subsequently found to be resistant by serial dilution studies $[15,16]$. Thus determination of sensitivity by disc diffusion is generally not recommended $[3,15]$. However, in one study a good correlation was found between agar dilution and disc diffusion methods for piperacillin, ceftazidime and ciprofloxacin against $E$. meningoseptica and disc diffusion breakpoints were proposed [17]. In many developing countries, it is often not possible to promptly determine minimum inhibitory concentration (MIC) by broth or agar dilution to most antibiotics because standard susceptibility testing powders from manufacturers may not be readily available. Although the E-test provides an easier alternative to determine the MIC, many laboratories can afford to keep only a stock of strips of the most commonly used antibiotics such as penicillin, ceftriaxone and ciprofloxacin, and not the ones needed to treat $E$. meningospetica meningitis as they are rarely needed. Moreover, for some antibiotics, important discrepancies in the MIC results have been noted between broth microdilution and E-test or agar dilution [13]. It is also unclear to what extent in vitro sensitivity, even by recommended methods, predicts clinical outcome when infections by Elizabethkingia sp. are encountered [13]. When the first cases occurred in our outbreak, a prompt decision had to be taken regarding antimicrobial treatment, and following discussion between the microbiologist and the paediatricians, it was decided to choose piperacillin because of its in vitro sensitivity by disc diffusion, its availability in the hospital, its in vitro susceptibility by broth microdilution and agar dilution in published studies, and its clinical effectiveness in a previous report [17-19]. Intravenous vancomycin was not chosen because of its limited penetration across the blood-brain barrier and because vancomycin assays were not available in Mauritius, thus making it almost impossible to optimize the dosage. Furthermore, we were encouraged by the rapid clinical response with piperacillin. We added rifampicin because of its in vitro sensitivity and because of many reports of successful treatment of E. meningoseptica infection with rifampicin adjuvant therapy $[4,20]$.

Prematurity is the primary host factor associated with neonatal infection. In two reviews of $E$. meningoseptica infections published in 1980 and 1997, prematurity based on gestation age or birthweight $<2,500 \mathrm{~g}$ was noted in $51 \%$ of 41 cases and $56 \%$ of 84 cases of neonatal meningitis respectively $[3,21]$. In the series reviewed by Bloch et $a l$., the mean age at the time of diagnosis was 12 days (median: 8 days) [3]. We observed similar features during our outbreak as seven of eight of our cases had a birth weight of $<2,500 \mathrm{~g}$ and the mean age at the time of diagnosis was 10 days.

In the medical literature, E. meningoseptica neonatal meningitis has been associated with a poor outcome. In one review, the mortality rate was $57 \%$ and hydrocephalus developed in $69 \%$ of survivors [3]. Deafness and developmental delay was also noted in $8 \%$ and $6 \%$ of cases respectively [3]. The poor outcome has historically been attributed to the unusual antibiotic susceptibility patterns of the organism and resistance to usual empirical antibiotic therapy, thus resulting in delays in instituting effective treatment. All seven babies treated in our outbreak survived the episode of meningitis, but one subsequently died from complications of hydrocephalus. Two other babies suffered complications and four recovered fully. The outcome was better than would have been expected from the 
medical literature and could have been due to the prompt initiation of effective antimicrobial treatment. However, we cannot exclude the possibility that the outbreak was caused by a less pathogenic strain as we did not serotype our isolates and there has been a suggestion that E. meningoseptica serotype $\mathrm{C}$ may predispose to hydrocephalus [4]. Nevertheless, in view of our results, the combination of piperacillin with rifampicin should be considered as a possible option for the treatment of E. meningoseptica neonatal meningitis if the isolate is susceptible in vitro. It is unlikely that a comparative trial of various treatment options of E. meningoseptica neonatal meningitis will ever be conducted because of the rarity of the condition and treatment recommendations will have to be based primarily on information from cases reported. Previously reported cases of treatment with piperacillin have led to relatively favourable outcome. In a review of 11 cases of E. meningoseptica meningitis from Taiwan, out of six cases treated with piperacillin, only one died but three developed hydrocephalus, and as the outcome was worse in the five cases treated with other antibiotics, the authors concluded that piperacillin was proved to be the drug of choice [19]. In Norway, two neonates with E. meningoseptica meningitis were treated with piperacillin and clindamycin, and both survived but developed hydrocephalus [22]. There is also a report of the successful use of piperacillin/tazobactam to treat one case of neonatal meningitis [4]. However, the in vitro antibiotic susceptibility pattern should also be taken into account when determining the most appropriate antimicrobial agent.

Historically, many cases of E. meningoseptica neonatal meningitis were reported from tropical countries and, in a study conducted in Cameroon in 1989, E. meningoseptica was the second commonest cause of meningitis in infants and children in that country [9,23-25]. In the last decade, a disproportionate number of cases of $E$. meningoseptica infections have been reported from Turkey and Taiwan, two relatively affluent countries with warm climates [5,18,26-29]. The reasons for this are unclear. It is conceivable that because of its resistance to multiple antibiotics, outbreaks of $E$. meningoseptica would be most likely to occur in places where broad-spectrum antibiotics are readily available and widely used but where infection control practice may be suboptimal. E. meningoseptica is generally resistant to antibiotics such as carbapenems and colistin which are increasingly used to treat extended-spectrum beta-lactamase-producing Klebsiella pneumoniae and multi-drug resistant Acinetobacter baumannii in countries where such organisms are increasingly encountered, and these characteristics may give it a survival advantage. Since the natural reservoir of E. meningoseptica is thought to be soil and water, the organism would also be expected to multiply more readily in warmer countries. The role of insects in the transmission of $E$. meningoseptica to humans has not been studied but may warrant further investigation as $E$. meningoseptica has been found to be one of the commonest bacteria cultured from the mid-gut of Anopheles gambiae mosquitoes [30].

E. meningoseptica is likely to be encountered more frequently in future because of its resistance to multiple antibiotics. Although many isolates are not clinically significant, it can sometimes cause serious infections. It is therefore essential that laboratories have the ability to identify the organism correctly. Good communication between clinicians and laboratory staff is also important for the management of infected patients. Finally neonatal meningitis caused by E. meningoseptica should be diagnosed and promptly treated with appropriate antibiotics to reduce risk of mortality and of neurological sequelae.

\section{References}

1. Steinberg JP and Burd EM (2010) Other gram-negative and gram-variable bacilli. In Mandell GL, Bennett JE, Dolin R, editors. Principles and Practice of Infectious Diseases. Philadelphia: Churchill Livingstone Elsevier. 3015-3033.

2. King EO (1959) Studies on a group of previously unclassified bacteria associated with meningitis in infants. Am J Clin Pathol 31: 241-247.

3. Bloch KC, Nadarajah R, Jacobs R (1997) Chryseobacterium meningosepticum: an emerging pathogen among immunocompromised adults. Report of 6 cases and literature review. Medicine (Baltimore). 76: 30-41.

4. Hoque SN, Graham J, Kaufmann ME, Tabaqchali S (2001) Chryseobacterium (Flavobacterium) meningosepticum outbreak associated with colonization of water taps in a neonatal intensive care unit. J Hosp Infect 47: 188-192.

5. Güngör S, Ozen M, Akinci A, Durmaz R (2003) A Chryseobacterium meningosepticum outbreak in a neonatal ward. Infect Control Hosp Epidemiol 24: 613-617.

6. Ministry of Health and Quality of Life (2004) Health statistics annual 2003: Deliveries performed in general hospitals 1992-2003. Available: http://www.gov.mu/portal/goc/moh/file/dig23.pdf. Accessed 10 January 2011.

7. Report of the working party on antibiotic sensitivity testing of the British Society for Antimicrobial Chemotherapy (1991). A guide to sensitivity testing: diffusion methods. J Antimicrob Chemother 27(Suppl D): 35-43.

8. Maraki S, Scoulica E, Manoura A, Papageorgiou N, Giannakopoulou C, Galanakis E (2009) A 
Chryseobacterium meningosepticum colonization outbreak in a neonatal intensive care unit. Eur J Clin Microbiol Infect Dis 28: 1415-1419.

9. Thong ML, Puthucheary SD, Lee EL (1981) Flavobacterium meningosepticum infection: an epidemiological study in a newborn nursery. J Clin Pathol 34: 429-433.

10. Bruun B, Jensen ET, Lundstrøm K, Andersen GE (1989) Flavobacterium meningosepticum infection in a neonatal ward. Eur J Clin Microbiol Infect Dis 8: 509-514.

11. George RM, Cochran CP, Wheeler WE (1961) Epidemic meningitis of the newborn caused by flavobacteria. II. Clinical manifestations and treatment. Am J Dis Child 101: 296-304.

12. Di Pentima MC, Mason EO Jr, Kaplan SL (1998) In vitro antibiotic synergy against Flavobacterium meningosepticum: implications for therapeutic options. Clin Infect Dis 26: 1169-1176

13. Fraser SL and Jorgensen JH (1997) Reappraisal of the antimicrobial susceptibilities of Chryseobacterium and Flavobacterium species and methods for reliable susceptibility testing. Antimicrob Agents Chemother 41: 2738-2741.

14. Kirby JT, Sader HS, Walsh TR, Jones RN (2004) Antimicrobial susceptibility and epidemiology of a worldwide collection of Chryseobacterium spp: report from the SENTRY Antimicrobial Surveillance Program (19972001). J Clin Microbiol 42: 445-448.

15. Aber RC, Wennersten C, Moellering RC Jr (1978) Antimicrobial susceptibility of flavobacteria. Antimicrob Agents Chemother 14: 483-487.

16. Johny M, Khuffash FA, Elhag KM (1983) Antimicrobial treatment of Flavobacterium meningosepticum infection. Ann Trop Paediatr 3: 125-128.

17. Chang JC, Hsueh PR, Wu JJ, Ho SW, Hsieh WC, Luh KT (1997) Antimicrobial susceptibility of flavobacteria as determined by agar dilution and disk diffusion methods. Antimicrob Agents Chemother 41:1301-1306.

18. Chiu $\mathrm{CH}$, Waddingdon M, Greenberg D, Schreckenberger PC, Carnahan AM (2000) Atypical Chryseobacterium meningosepticum and meningitis and sepsis in newborns and the immunocompromised, Taiwan. Emerg Infect Dis 6: 481486.

19. Lin CH, Huang FY (1991) Clinical observation of neonatal meningitis caused by Flavobacterium meningosepticum. Zhonghua Min Guo Xiao Er Ke Yi Xue Hui Za Zhi 32: 171176. Chinese

20. Lee EL, Robinson MJ, Thong ML, Puthucheary SD (1976) Rifamycin in neonatal flavobacteria meningitis. Arch Dis Child 51: 209-213.

21. Dooley JR, Nims LJ, Lipp VH, Beard A, Delaney LT (1980) Meningitis of infants caused by Flavobacterium meningosepticum: report of a patient and analysis of 63 infections. J Trop Pediatr 26: 24-30.

22. Abrahamsen TG, Finne PH, Lingaas E (1989) Flavobacterium meningosepticum infections in a neonatal intensive care unit. Acta Paediatr Scand 78: 51-55.

23. Madruga M, Zanon U, Pereira GM, Galvão AC (1970) Meningitis caused by Flavobacterium meningosepticum. The first epidemic outbreak of meningitis in the newborn in South America. J Infect Dis 121: 328-330.

24. Lapage SP, Owen RJ (1973) Flavobacterium meningosepticum from cases of meningitis in Botswana and England. J Clin Pathol 26: 747-749.

25. Kokindombo PO, Nko'o Amvenne S, Ndjitoyap Ndam EC, Wouaffo Ndayo M, Tangam Ouambo T, Tietche F, Foumbi J, Borderon JC, Laugier J, Mbede J (1991) Purulent meningitis due to Flavobacterium meningosepticum in Cameroonian children. Ann Pediatr (Paris) 38: 491-495.

26. Tekerekoglu MS, Durmaz R, Ayan M, Cizmeci Z, Akinci A (2003) Analysis of an outbreak due to Chryseobacterium meningosepticum in a neonatal intensive care unit. New Microbiol 26: 57-63.

27. Ceyhan M, Yildirim I, Tekeli A, Yurdakok M, Us E, Altun B, Kutluk T, Cengiz AB, Gurbuz V, Barin C, Bagdat A, Cetinkaya D, Gur D, Tuncel O (2008). A Chryseobacterium meningosepticum outbreak observed in 3 clusters involving both neonatal and non-neonatal pediatric patients. Am J Infect Control 36: 453-457.

28. Lin YT, Chiu CH, Chan YJ, Lin ML, Yu KW, Wang FD, Liu CY (2009) Clinical and microbiological analysis of Elizabethkingia meningoseptica bacteremia in adult patients in Taiwan. Scand J Infect Dis 41: 628-634.

29. Wang JL, Chen ML, Lin YE, Chang SC, Chen YC (2009) Association between contaminated faucets and colonization or infection by nonfermenting gram-negative bacteria in intensive care units in Taiwan. J Clin Microbiol 47: 32263230 .

30. Dong Y, Manfredini F, Dimopoulos G (2009) Implication of the mosquito midgut microbiota in the defense against malaria parasites. PLoS Pathog 5: e1000423.

\section{Corresponding author}

Dr. M. I. Issack

Central Health Laboratory

Victoria Hospital

Candos, Mauritius

Telephone: +2304270531

Fax: + 2304245848

Email address: missack@intnet.mu

Conflict of interests: No conflict of interests is declared. 